Ann. Biol. anim. Bioch. Biophys., 1979, 19 (2A), 385-391.

\title{
Absence de transformation bactérienne des acides biliaires chez la truite arc-en-ciel, Salmo gairdneri, élevée à $16^{\circ} \mathrm{C}$
}

par E. SACQUET, R. LESEL, C. MEJEAN, M. RIOTTOT, C. LEPRINCE

Laboratoire des Animaux sans Germes du C.N.R.S. I.N.R.A. 78350 Jouy-en-Josas,

Laboratoire des microorganismes, I.N.R.A., Saint-Pée sur Nivelle, 64310 Ascoin.

Summary. Absence of bacterial conversion of bile acids in the rainbow trout, Salmo gairdneri, raised af $16^{\circ} \mathrm{C}$.

The fecal bile acids of the rainbow trout, Salmo gairdneri, raised at $16^{\circ} \mathrm{C}$, were entirely conjugated. These bile acids were cholic acid, chenodeoxycholic acid and trace amounts of $3 \alpha, 12 \alpha$-dihydroxy-7-ceto and $3 \alpha$-hydroxy-7-ceto-5 $\beta$-cholanoic acids. Therefore, there was very little bacterial conversion of bile acids in that animal species. This observation favorized the absence of a bacterial flora adapted to the digestive fract of that fish.

II n'existe qu'un nombre restreint d'informations concernant la flore bactérienne présente dans le tractus digestif des poissons, (Horsley, 1977) et en particulier des Salmonidés d'élevage (Trust, 1975). Chez les poissons qui vivent dans des eaux dont la température est voisine de $15^{\circ} \mathrm{C}$, les examens bactériologiques mettent en évidence un nombre de bactéries qui, comparé à celui qui est observé chez la plupart des homéothermes, est extrêmement faible; ce nombre varie de $10^{2}$ à $10^{8} / \mathrm{g}$ de fèces chez les premiers (Trust et Sparrow, 1974 ; Trust, 1975, Horsley, 1977), de $10^{10}$ à $10^{11}$ chez les seconds (Ducluzeau, 1969). Il n'est pas certain que ce faible nombre de bactéries constitue une flore bactérienne propre à la truite, plutôt qu'une flore de passage, c'est-à-dire propre au milieu extérieur dans lequel vit cet animal. Il est douteux que cette flore soit capable d'exercer des transformations métaboliques (Sedalian, 1968 ; Colin, 1972 ; Prejs ef Blaszyk, 1977).

Dans le présent travail, nous recherchons la présence des métabolites bactériens des acides biliaires dans la bile ef dans les fèces de la truite arc-en-ciel, parce que les transformations, que fait subir à ces espèces chimiques la flore bactérienne chez les sujets homéothermes, sont d'une telle ampleur que la mise en évidence des métabolites ainsi formés, constitue un test biochimique très sensible de l'activité métabolique de la flore bactérienne du tractus digestif. 


\section{Matériel ef méthodes.}

Sujets d'expérience et prélèvements. - Les sujets sont des truitelles âgées de huit mois environ, acclimatées depuis un mois à une température de $16 \pm 1{ }^{\circ} \mathrm{C}$. Après cette période d'acclimatation les truites sont placées dans des bacs pourvus d'un dispositif qui permet de recueillir les fèces. Ces bacs, d'une capacité de $20 \mathrm{I}$, reçoivent par minute, 1 I d'eau qui provient du circuit de distribution urbaine. Les truites sont accoutumées à ces bacs pendant une semaine. Les fèces sont ensuite collectées pendant quinze jours. Le dispositif de récolte dont sont pourvus ces bacs (fig. 1) est constitué d'un tapis roulant à mailles larges, qui retiennent les fèces émises, tout en laissant passer l'eau. Au bout de la zone de collecte, et afin d'éviter toute altération bactérienne postérieure à leur émission, un jet d'air comprimé projette les fèces dans de l'éthanol. Le temps écoulé entre émission et fixation des fèces est inférieur à une minute.

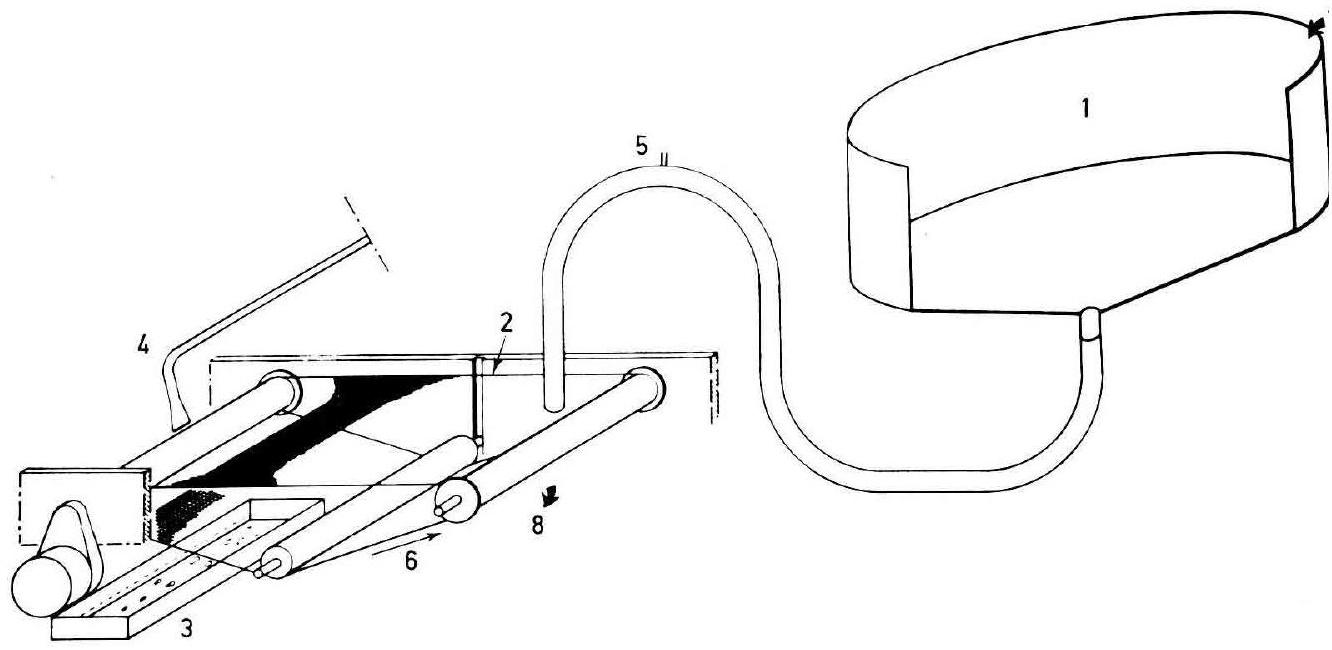

FIG. 1. - Appareil de collecte des fèces de poisson. 1 : bac-entonnoir hébergeant la truite. 2 : tapis collecteur à grandes mailles. 3 : bac de collecte et fixation des fèces. 4 : arrivée d'air sous pression. 5 : régulateur du niveau d'eau dans le bac. 6 : sens de rotation du tapis. 7 : arrivée d'eau. 8 : évacuation d'eau après passage au travers du tapis.

Au cours des périodes d'acclimation et pendant toute la durée de l'expérimentation, les truites reçoivent, deux fois par jour, un aliment commercial de composition inconnue, qui contient, au maximum, 11 p. 100 d'humidité, 5 p. 100 de cellulose, 14 p. 100 de matières minérales ef au minimum, 45 p. 100 de matières protéiques brutes et 7 p. 100 de matières grasses.

Une dizaine de vésicules biliaires sont prélevées sur des truites qui proviennent du même lot que les truites dont les fèces sont recueillies. Ces vésicules sont conservées dans de l'éthanol absolu jusqu'au moment de l'analyse.

Techniques biochimiques. - La plupart de ces techniques ont été décrites précédemment (Sacquet ef al., 1975). Elles seront, ici, brièvement exposées. 
- Extraction des acides biliaires. Les vésicules biliaires sont broyées dans de l'éthanol et extraites par ébullition à reflux pendant une heure dans ce solvant. Les extraits éthanoliques sont centrifugés et le surnageant est recueilli. Les matières fécales, également broyées dans l'éthanol, sont extraites par ce solvant au Kumagawa pendant $48 \mathrm{~h}$. Les extraits ainsi obtenus servent à deux recherches : la recherche des acides biliaires libres et l'analyse des diverses espèces chimiques d'acides biliaires ; celle-ci est ou non précédée d'une réaction de solvolyse.

- Recherche des acides biliaires libres. Les extraits éthanoliques sont chromatographiés, en présence de standards d'acides biliaires libres et conjugués, sur couche mince de gel de silice $G$ à l'aide du mélange de solvants : acide propionique/acétate d'isoamyle/propanol/eau : 15/20/10/5, v/v (Hofmann, 1963). Nous appellerons ce système, système $A$. Les chromatogrammes sont révélés par l'acide sulfurique dilué au $1 / 3$, chauffage pendant $15 \mathrm{~min}$. à $110^{\circ} \mathrm{C}$ et examen à $360 \mathrm{~nm}$. Ce test, très sensible, permet de déceler des acides biliaires qui existent à l'état de traces el de distinguer les acides biliaires conjugués à la taurine ou à la glycine, des acides biliaires libres.

- Analyse des diverses espèces chimiques d'acides biliaires. Les acides biliaires contenus dans l'extrait éthanolique sont séparés des stérols neutres par la méthode de Grundy et al. (1965). Celle-ci consiste d̀ effectuer d'abord une hydrolyse alcaline des esters du cholestérol eł à extraire celui-ci par l'éther de pétrole. Une hydrolyse alcaline plus poussée libère les acides biliaires de leur liaison avec la taurine ef la glycine. Après acidification à $\mathrm{pH} 2$, les acides biliaires sont extraits par le chloroforme. L'extrait est mis à $\mathrm{sec}$, repris par le méthanol et méthylé par le diazométhane. Les esters méthyliques ainsi obtenus font l'objet de deux types de chromatographie : chromatographie en couche mince ou CCM, chromatographie en phase gazeuse ou CPG.

La première est une chromatographie en couche mince de gel de silice $G$ de $60 \mathrm{~cm}$ de long et $0,5 \mathrm{~mm}$ d'épaisseur à l'aide du mélange chloroforme/acétone/ méthanol : 70/25/5, v/v (Sacquet ef al., 1975), que nous appellerons système B. Elle est faite en présence de standards, placés de chaque côté de l'échantillon analysé, qui sont révélés par l'acide sulfurique comme cela a été précédemment indiqué. ll est possible de préserver de l'action de l'acide sulfurique les échantillons analysés et de ne révéler que les standards. Ce procédé permet de localiser avec précision les surfaces de gel à prélever, en dépit des variations propres à ce type de chromatographie.

La chromatographie en phase gazeuse se fait après silylation des esters méthyliques des acides biliaires, sur colonne en verre remplie de 3 p. 100 d'OV 17 fixé sur Gaschrom $Q$ selon la technique de Siegfried et al. (1975). Sont soumis à cette CPG, soit les extraits méthanoliques méthylés et silylés obtenus comme il est ci-dessus indiqué, soit les éluats obtenus par action de l'acétone sur les prélèvements de gel de silice $G$ provenant de la chromatographie en couche mince.

Le tableau 1 indique les séparations obtenues par ces deux méthodes. Leur combinaison permet d'obłenir des informations très complètes : c'est ainsi que les dérivés des acides désoxycholique et chénodésoxycholique, qui ne sont pas séparés en CPG, sont bien séparés en CCM. II en est de même pour les acides cholique et lithocholique. Les 3 -céto-12 $\alpha$-hydroxy et 3-céto-7 $\alpha, 12 \alpha$-dihydroxy-5 $\beta$-cholanoates de méthyle qui chevauchent en CPG sont bien séparés en CCM. La présence de petites 
quantités d'acides biliaires porteurs de fonction cétone qui risquent de ne pas être détectés en CCM sont mis en évidence en CPG.

\section{TABLE 1}

Chromatographie des acides cholique et chénodésoxycholique ainsi que de leurs principaux métabolites bactériens

\begin{tabular}{|c|c|c|}
\hline & CPG $\left({ }^{1}\right)$ & $\operatorname{CCM}\left({ }^{2}\right)$ \\
\hline 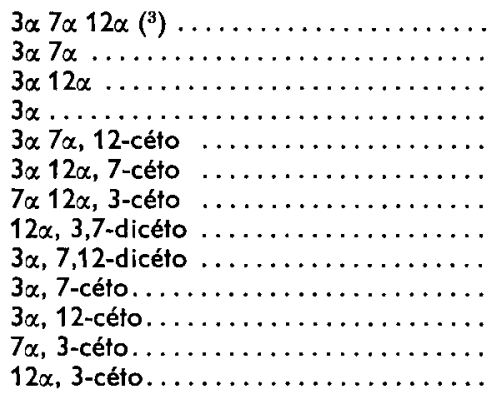 & $\begin{array}{l}0,90 \\
1,00 \\
1,00 \\
0,93 \\
1,97 \\
2,03 \\
1,68 \\
3,10 \\
3,38 \\
1,98 \\
1,91 \\
1,84 \\
1,67\end{array}$ & $\begin{array}{l}0,40 \\
1,00 \\
1,08 \\
1,44 \\
0,88 \\
0,88 \\
1,04 \\
\text { n.d. }\left({ }^{4}\right) \\
\text { n.d. }\left(^{4}\right) \\
1,30 \\
1,25 \\
1,44 \\
1,44\end{array}$ \\
\hline
\end{tabular}

(1) CPG : chromatographie en phase gazeuse (Siegfried ef al., 1975) : temps de rétention par rapport au dérivé de l'acide chénodésoxycholique.

(2) CCM : chromatographie en couche mince (Sacquet et al., 1975) : position par rapport au chénodésoxycholate de méthyle.

$\left({ }^{3}\right)$ La lettre $\alpha$ indique la présence d'un hydroxyle en position $\alpha$.

(4) n.d. : non déterminé ; position voisine du lithocholate de méthyle.

- Solvolyse. Un échantillon d'extrait éthanolique de fèces a été solvolysé avant d'être soumis à la technique d'analyse que nous venons de décrire. La solvolyse est faite en éliminant d'abord les minéraux par passage sur XAD-2 et en faisant agir pendant 18 heures une solution anhydre de méthanol/acétone/ $\mathrm{HCl} 12 \mathrm{~N}: 1 / 9 / 0,075$, $\mathrm{v} / \mathrm{v}$.

\section{Résultats.}

L'examen des chromatogrammes faits, soit à partir de la bile, soit à partir des fèces, dans le système $A$ montre deux taches ayant la position de l'acide taurocholique $(\mathrm{RF}=0,07)$ et celle des acides taurochénodésoxycholique ou taurodésoxycholique $(R F=0,16)$. Il n'y a aucune trace d'acides libres. Après hydrolyse alcaline et méthylation, la chromatographie dans le système B permet de préciser la présence de cholate et de chénodésoxycholate de méthyle ainsi que l'absence de désoxycholate de méthyle. La chromatographie en phase gazeuse confirme la présence de ces deux acides biliaires dans la proportion molaire de 90 p. 100 d'acide cholique pour 10 p. 100 d'acide chénodésoxycholique dans la bile, 68 p. 100 du premier pour 32 p. 100 du second dans les fèces. Les chromatogrammes faits à partir des fèces révèlent, en 
plus des pics correspondant aux acides cholique et chénodésoxycholique, la présence d'espèces chimiques non séparées, qui recouvrent une surface dont les temps de rétention par rapport à l'acide chénodésoxycholique s'étendent de 1,70 à 2,45 (fig. 2). Ces

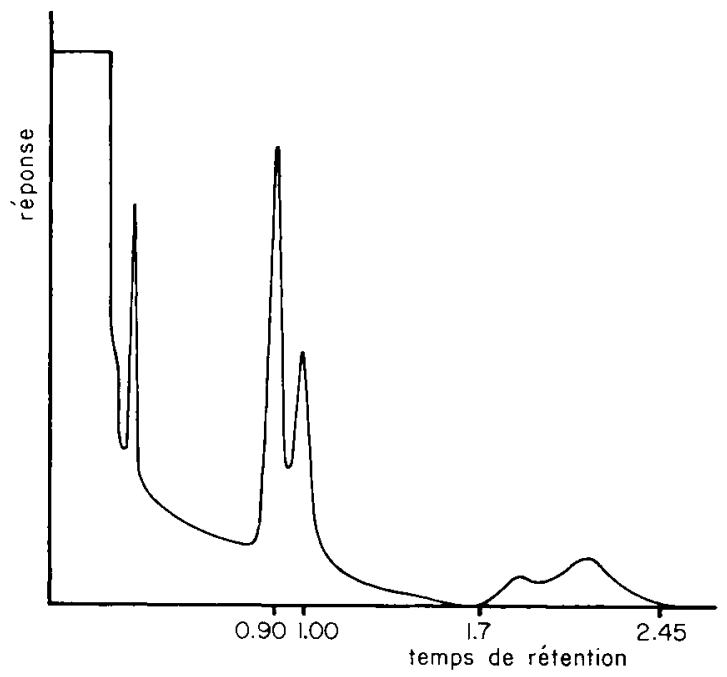

FIG. 2. - Chromatographie en phase gazeuse faite à partir des extraits fécaux de truites. Temps de rétention par rapport au dérivé de l'acide chénodésoxycholique. Le premier pic en partant de la gauche est celui de l' $\alpha$-cholestane, témoin-interne, le deuxième pic correspond à l'acide cholique, le troisième à l'acide chénodésoxycholique, la surface comprise entre 1,70 et 2,45 correspond à des substances alimentaires non identifiées et à de petiłes quantités d'acides biliaires porteurs de fonction cétone.

substances pourraient donc être les métabolites bactériens des acides cholique et chénodésoxycholique suivants, dont les temps de rétention par rapport à l'acide chénodésoxycholique sont indiqués entre parenthèses : acide 3-céto-7 $\alpha$-hydroxy-5 $\beta$ cholanoïque $(1,84)$, $3 \alpha$-hydroxy-12-céto-5 $\beta$-cholanoïque $(1,91), 3 \alpha, 7 \alpha$-dihydroxy-12céto- $5 \beta$-cholanoïque $(1,97), 3 \alpha$-hydroxy-7-céto- $5 \beta$-cholanoïque $(1,98) 3 \alpha, 12 \alpha$-hydroxy7-céto-5 3 -cholanoïque $(2,03)$. Mais l'aliment extrait par l'éthanol au Kumagawa et traité de la même manière que les fèces, donne dans cette région du chromatogramme une image identique à celle qui est obtenue à partir des fèces, en sorte qu'il est impossible par le simple examen des chromatogrammes en CPG obtenus à partir des fèces, de savoir si l'on est en présence uniquement de ces substances alimentaires ou si celles-ci masquent de petites quantités des méłabolites bactériens ci-dessus énumérés. Pour lever ce doute, les chromatogrammes faits à partir des fèces en couche mince dans le système $B$ sont découpés en surfaces correspondant aux esters méthyliques de ces acides biliaires, et le gel de silice de ces différentes surfaces est collecté et élué par l'acétone. Comme la position de certains dérivés porteurs de fonction cétone n'est pas connue avec exactitude, le gel de silice correspondant à toutes les surfaces qui s'étendent depuis le chénodésoxycholate de méthyle jusqu'au front du chromatogramme est également collecté et élué. Les éluats sont silylés ef soumis individuellement à la chromatographie en phase gazeuse. Il n'apparaît alors que des traces cor- 
respondant aux acides $3 \alpha, 12 \alpha$-dihydroxy-7-céto et $3 \alpha$-hydroxy-7-céto-5 $\beta$-cholanoïque : moins de 1 p. 100 de l'ensemble des acides biliaires.

Enfin, après solvolyse, les chromatogrammes en CPG, ne sont pas modifiés en sorte que, ou bien les acides biliaires fécaux ne sont pas sulfatés ou bien l'acide cholique et l'acide chénodésoxycholique sont sulfatés dans les mêmes proportions.

\section{Discussion.}

Les acides biliaires primaires de la truite sont les acides taurocholique et faurochénodésoxycholique. Ces résultats s'accordent avec ceux de Denton et Yousef (1975) qui trouvent dans la bile des proportions semblables d'acide cholique (85 p. 100) et chénodésoxycholique (14 p. 100) chez des truites arc-en-ciel âgées de 6 à 13 mois. Ces auteurs trouvent, en outre, de petites quantités de ces acides biliaires conjugués à la glycine ou sulfatés.

Les proportions des acides cholique et chénodésoxycholique sont sensiblement différentes au niveau de la bile et des fèces puisqu'au niveau de la bile les proportions sont 90 p. 100 du prenier et 10 p. 100 du second et dans les fèces 65 p. 100 du premier et 35 p. 100 du second. Un fait semblable existe chez le rat axénique, animal dépourvu de vésicule biliaire, chez lequel, au niveau de l'intestin grêle l'acide cholique constitue 38 p. 100 de l'ensemble des acides biliaires el au niveau des fèces, 25 p. 100 (Sacquet et al. 1975). Cette différence s'explique par la plus grande vitesse d'absorption de l'acide taurocholique au niveau de la région terminale de l'intestin grêle. La participation de cet acide au cycle entéro-hépatique est ainsi plus grande que celle des autres acides biliaires. La truite ef le rat partageraient cette même propriété.

Le fait essentiel est l'absence presque complète de transformation bactérienne des acides biliaires chez la truite. Denton et Yousef n'avaient observé au niveau de la bile de Salmo gairdneri que des traces de cétones, moins de 1 p. 100 . Mais cela ne permet pas de présumer de la quantité qui existe au niveau des fèces, parce que nous ignorons dans quelle mesure le foie de la truite réduit les cétones, comme le fait si bien le foie du rat.

La comparaison entre le rat et la truite se prête à d'autres remarques. Chez le premier, les acides biliaires sont entièrement conjugués dans la bile et entièrement libres dans les fèces. Les fèces du rat contiennent au plus 5 p. 100 des acides biliaires primaires du rat, c'est-à-dire de ceux qui sont initialement synthétisés par l'hépatocyte et qui sont seuls présents chez le rat axénique, et 95 p. 100 de métabolites bactériens (Sacquet et al., 1975). Dans les fèces de la truite les acides biliaires demeurent entièrement conjugués et ne subissent à peu près aucune transformation décelable par les techniques que nous avons mises en œuvre.

A l'inverse du rat, la truite ne possède donc pas au niveau de son tractus digestif une flore bactérienne capable de modifier le métabolisme des sels biliaires. Ceci constitue un argument pour soutenir l'absence, chez cette espèce animale, de microflore que nous appellerons « endogène », c'est-à-dire l'absence d'espèces bactériennes qui se seraient adaptées à vivre au niveau du tractus digestif de la truite et à utiliser à leur profit des substances issues de son métabolisme. La truite ne possèderait, au mieux, qu'une flore « exogène » une flore de passage, qui pourrait être capable de modifier des substances provenant du milieu extérieur, en particulier certaines subs- 
tances alimentaires, dans la mesure où celles-ci séjourneraient dans ce milieu dans un état où elles seraient accessibles à ces microorganismes. Paris ef al. (1977) ont mis en évidence la présence d'une faible quantité d'acides gras volatils au niveau du tractus digestif de la truite ainsi que de la carpe commune (Cyprinus carpio) et de la carpe amour (Ctenopharyngodon idella).

Enfin, la truite n'est vraisemblablement pas la seule espèce animale qui présente les caractéristiques ci-dessus décriłes, car de nombreux poïkilothermes qui vivent dans les eaux froides présentent des flores bactériennes intestinales apparemment très paurres (Lesel et Menet, 1977 ; Mac Bee, 1960). Chez de tels animaux l'examen des acides biliaires fécaux serait un complément utile des études bactériologiques de la microflore du tractus digestif.

Reçu en mai 1978.

Accepté en octobre 1978.

\section{Références}

COLIN D. A., 1972. Relations entre la nature de l'alimentation et l'importance de l'activité chitinolytique du tube digestif de quelques téléostéens marins. C. R. Soc. Biol., 166, 95-98.

DENTON J. E., YOUSEF M. K., 1975. Bile acid composition of rainbow trout, Salmo gairdneri. Lipids, 9, 945-950.

DUCLUZEAU R., 1969. Influence de l'espèce zoologique sur la microflore du tracłus gastro-intestinal. Rev. Immunol., 33, 345-384.

GRUNDY S. M., AHRENS E. H., MIETTINEN T. A., 1965. Quantitative isolation and gas-liquid chromatographic analysis of total fecal bile acids. J. Lipid Res., 7, 397-410.

HOFMANN A. F., 1963. Thin layer absorption of free and conjugated bile acids on silicic acid. J. Lipid Res., 2, 127-128.

HORSLEY R. W., 1977. A review of the bacterial flora et teleosts and elasmobranchs, including methods for its analysis. J. Fish Biol., 10, 529-553.

LESEL R., MENET J. P., 1977. The bacterial microflora in the digestive tracts of penguins and fishes from the Kerguelen Islands, 569-578. In LLANO G. A., Adaptation within antarctic ecosystems. Proc. 3rd SCAR Symp. on antarctic biology, Washington, U. S. A.

MC BEE R. H., 1960. Intestinal flora of some antarctic birds and mammals. J. Bacteriol., 79, 311-312.

PARIS H., MURAT J. C., CASTILLA C., 1977. Etude des acides gras volatils dans l'intestin de trois espèces de poissons Téléostéens. C. R. Soc. Biol., 171, 1297-1300.

PREJS A., BLASZYK M., 1977. Relationships between food and cellulase activity in freshwater fishes. J. Fish Biol., 11, 447-452.

SACQUET E., van HEIJENOORT Y., RIOTTOT M., LEPRINCE C., 1975. Action de la flore microbienne du tractus digestif sur le métabolisme des acides biliaires chez le rat. Biochim. biophys. Acta, 380, 52-65.

SEDALIAN A., 1968. Recherche de germes chitinolytiques dans le tube digestif de quelques téléostéens marins. C. R. Soc. Biol., 162, 949-951.

SIEGFRIED C. M., DOISY E. A. Jr, ELLIOTT W. H., 1975. Bile acids XLIV. Quantitation of bile acids from the bile fistula rat given $\left({ }^{4-14} \mathrm{C}\right)$ cholesterol. Biochim. biophys. Acta, 380, 66-75.

TRUST T. J., 1975. Facultative anaerobie bacteria in the digestive tract of chum salmon (Oncorhynchus kefa) maintained in fresh water under defined culture conditions. Appl. Microbiol., 29, 663-668.

TRUST T. J., SPARROW R. A. H., 1974. The bacterial flora in the alimentary tract of freshwater salmonid fishes. Can. J. Microbiol., 20, 1219-1228. 